\title{
Munitionsaltlasten vor deutschen Küsten und deren Bezug zum Gewässerschutz-, Naturschutz- und Gefahrenabwehrrecht
}

\author{
Roman Kollenberg
}

(c) Der/die Autor(en) 2022. Dieser Artikel ist eine Open-Access-Publikation.

Insbesondere der Politikdiskurs in den norddeutschen Bundesländern, aber auch die Wissenschaft befasst sich schon seit einigen Jahren mit den Munitionsaltlasten, die sich vor den deutschen Küsten befinden. Dass diese als Gefahrenquellen mit drohenden erheblichen schädlichen Folgen für Mensch und Natur beseitigt werden müssen, erscheint immer dringlicher. Jedoch erweist sich der rechtliche Rahmen dieser Beseitigungsmaßnahmen vor dem Hintergrund der bestehenden gewässerschutz-, naturschutz- und gefahrenabwehrrechtlichen Regelungen als sehr unübersichtlich. Er soll daher in dem vorliegenden Beitrag ausgeleuchtet werden.

\section{Problematik}

In den Meeresschutzgebieten vor der Nord- und Ostsee befinden sich seit dem Ende des Ersten und Zweiten Weltkrieges zahlreiche Bomben ${ }^{1}$, Minen und andere Munitionstypen, die bei Kriegshandlungen, Manövern oder im Rahmen gezielter Entsorgungen im Meer versenkt wurden. ${ }^{2}$ Alle Munitionsfunde aus der deutschen Nord- und Ostsee werden bei der „Zentralen Meldestelle für Munition im Meer“ im Maritimen Sicherheitszentrum (MSZ) Cuxhaven registriert und veröffentlicht. So wurden im Jahr 2018 in allen deutschen Seegewässern insgesamt 376 Meldungen mit mehr als 3840 Objekten aufgenommen. ${ }^{3}$ Diese Meldungen werden unter anderem von der Polizei, aus der Wissenschaft ${ }^{4}$ oder privaten Unternehmen eingereicht. ${ }^{5}$ Insgesamt wird die Zahl der Munitionsaltlasten allein für die Nordund Ostsee auf rund 1,6 Mio. Tonnen konventionelle (meist Sprengmunition) und ca. 5000 Tonnen chemische Munition geschätzt. ${ }^{6}$ Während konventionelle Munition wie Sprengstoffe oder Brandmittel z. B. weißen Phosphor enthält und sich ihre Wirkung dementsprechend durch eine Detonation oder Inbrandsetzung entfaltet, zeichnet sich chemische $\mathrm{Mu}-$ nition durch eine Füllung mit chemischem Kampfstoff aus. Ihr Einsatzzweck ist nicht die Zerstörung von Infrastruktur, sondern vielmehr direkt eine vorübergehende oder endgültige Außergefechtsetzung von Menschen durch die vom Typ des Kampfstoffs abhängige Giftwirkung. ${ }^{7}$ Für eine lange Zeit galt nach Einschätzung der verantwortlichen Stellen, dass die Munitionsaltlasten im Meer verbleiben sollten. Grund dafür war, dass diese aus behördlicher Sicht bislang keine generelle, großräumige Gefährdung der Umwelt über die lokal betroffenen Flächen hinaus darstellten. ${ }^{8}$ Hinzu kommt, dass die Räumung solcher Sprengkörper sehr kostenintensiv ist. ${ }^{9}$ Die im Meer versenkte Munition zeigte sich in einem Zustand, der statistisch und qualitativ teilweise schwierig zu klassifizieren war und noch immer ist.

\subsection{Gefahrenpotential für Menschen und Meeresumwelt}

Besonders gefährdet ist der Schiffsverkehr und in diesem Zusammenhang auch Leib und Leben von Menschen. ${ }^{10}$ So kommen Gefährdungen etwa in Betracht, wenn die $\mathrm{Mu}-$ nition absichtlich (z. B. zwecks Untersuchungen) oder un-

Cand jur. Roman Kollenberg,

Studentische Hilfskraft am Lehrstuhl für Öffentliches Recht,

insbesondere Umweltrecht, und Verfassungsgeschichte

(Prof. Dr. Michael Kotulla, M.A.), Universität Bielefeld,

Bielefeld, Deutschland absichtlich (z.B. durch Fischerei mit Grundschleppnetzen) gehoben wird und daraufhin möglicherweise detoniert. ${ }^{11}$ Eine Gefahr besteht auch in den Fällen, in denen die Munition oder die darin enthaltenen Wirkmittel (z. B. Phosphor oder Senfgas) durch Meeresströmungen an die Küsten gelangen und dort unsachgemäß behandelt werden; so beispielsweise bei direktem Kontakt mit den schädlichen Inhaltsstoffen durch Personen, die Steine oder Muscheln am Strand sammeln. ${ }^{12}$ In Betracht kommt aber auch ein indirekter Kontakt mit toxischen Substanzen durch den Verzehr kontaminierter Meeresprodukte (z.B. Fisch). ${ }^{13}$ Die

1) Arndt/Jung, Die Deutschen Sperrbrecher, 1914-1945. Konstruktion - Ausrüstung - Bewaffnung - Aufgaben - Einsatz, 1979, S. 197.

2) BT-Drs. 14/7277, S. 2.

3) Bund/Länder-Ausschuss Nord- und Ostsee (BLANO): Munitionsbelastung der deutschen Meeresgewässer - Entwicklungen und Fortschritt: Jahresbericht 2018, Stand 21.9.2021, abrufbar unter https://www.schleswig-holstein.de/DE/UXO/Berichte/PDF/Berichte/ag_blano_fortschritt2018.pdf?__blob=publicationFile \&v= 2, S. 6.

4) So hat sich im Rahmen der Erforschung und Beseitigung von Munitionsaltlasten die Bund-/Länder-Arbeitsgemeinschaft: Nord- und Ostsee (BLANO) - Expertenkreis Munition im Meer gebildet, welche regelmäßig über Fortschritte, Erkenntnisse und Empfehlungen berichtet. Alle Berichte werden auf dem vom Land Schleswig-Holstein betriebenen Internet-Portal „Munitionsbelastung der deutschen Meeresgewässer" (www.munitionim-meer.de) öffentlich zur Verfügung gestellt.

5) Bund/Länder-Ausschuss Nord- und Ostsee (BLANO): Munitionsbelastung der deutschen Meeresgewässer - Entwicklungen und Fortschritt: Jahresbericht 2018, Stand 21.9.2021, abrufbar unter https://www.schleswig-holstein.de/DE/UXO/Berichte/ $\mathrm{PDF} /$ Berichte/ag_blano_fortschritt2018.pdf?__blob=publicationFile $\& v=2$, S. 6 .

6) Umwelt Bundesamt (UBA): Munition im Meer, Stand 21. 9. 2021, abrufbar unter https://www.umweltbundesamt.de/themen/ wasser/meere/nutzung-belastungen/munition-im-meer\#schadstoffbelastung-durch-konventionelle-munition.

7) Bund/Länder-Ausschuss Nord- und Ostsee (BLANO): Munitionsbelastung der deutschen Meeresgewässer - Entwicklungen und Fortschritt: Jahresbericht 2018, Stand 21.9.2021, abrufbar unter https://www.schleswig-holstein.de/DE/UXO/Berichte/ PDF/Berichte/ag_blano_fortschritt2018.pdf?__blob=publicationFile \&v=2, S. 7.

8) BT-Drs. 14/7277, S. 2.

9) Jenisch, NordÖR 2020, 493, 496, der die Räumung eines einzigen Sprengkörpers auf See mit durchschnittlich 30000 Euro beziffert.

10) Antwort auf die Kleine Anfrage ,Sprengung von Munitionslasten und Kampfmitteln in Meeresschutzgebieten", BTDrs. 19/15325, S. 2 .

11) Bund/Länder-Ausschuss Nord- und Ostsee (BLANO): Munitionsbelastung der deutschen Meeresgewässer - Entwicklungen und Fortschritt: Jahresbericht 2018, Stand 21.9.2021, abrufbar unter https://www.schleswig-holstein.de/DE/UXO/Berichte/ PDF/Berichte/ag_blano_fortschritt2018.pdf?__blob=publicationFile $\& v=2$, S. 14 .

12) Der Spiegel: Vermeintlicher Bernstein verbrennt Strandbesucher, Stand 21.9.2021, abrufbar unter: https://www.spiegel.de/wissenschaft/mensch/phosphor-aehnelt-bernstein-und-verbrenntmann-am-strand-a-943631.html.

13) Koske/Straumer, Wissenschaft erleben 2/2018, 10. 
Möglichkeit mit Munitionsaltlasten in Kontakt zu kommen, nimmt gerade durch die wachsende wirtschaftliche Nutzung des Meeresbodens - wie etwa durch den Bau von Offshore-Windparks zur Windenergiegewinnung auf hoher See und deren Netzanbindung - zu und stellt somit eine stetig steigende Gefahr für das dortige Arbeitspersonal dar. ${ }^{14}$ Als schwerwiegende Gefahr erweist sich die Munitionsaltlast nicht zuletzt aber auch für die Flora und Fauna der Meeresumwelt. Forscher des Thünen-Instituts für Fischereiökologie entdeckten im Rahmen des Projektes DAIMON (Decision Aid for Marine Munitions) Sprengstoffrückstände wie TNT (Trinitrotoluol) bei der Untersuchung von heimischen Fischarten wie der Kliesche (Limanda limanda), aber auch in diversen bodenlebenden Arten und experimentell exponierten Miesmuscheln. ${ }^{15}$ So konnten bei $25 \%$ der Klieschen aus dem Versenkungsgebiet Lebertumore festgestellt werden, wohingegen die Tumorrate bei Klieschen aus einem unbelasteten Gebiet bei nur knapp $5 \%$ lag. ${ }^{16}$ Ein Zusammenhang der Tumorerkrankungen mit dem TNT wird von den Forschern als nicht abwegig eingestuft. Auf den freiliegenden Munitionsaltlasten halten sich zudem Muscheln, Seesterne und Algen auf, welche unweigerlich toxische Substanzen aufnehmen können. ${ }^{17}$

\subsection{Sprengungen als Methode der Beseitigung}

Zunächst könnte als taugliche Beseitigungsmaßnahme die Bergung der Munitionsaltlast in Betracht kommen. Jedoch spricht in einigen Fällen gegen diese Form der Beseitigung, dass die Munition im Laufe der Zeit sehr stark korrodiert ist und dass die Hüllen der Munitionskörper sehr dünnwandig, beschädigt oder gar nicht mehr vorhanden sind..$^{18}$ Ebenso sind auch die Zündeinrichtungen der Munitionsaltlasten betroffen. Einige der Zünder sind auch heute noch in einem funktionsfähigen Zustand und können die Ladung zur Explosion bringen, wohingegen andere Zündsysteme ihre Funktionalität verloren haben, nachdem sie dem Korrosionsvorgang erlegen sind. Der aufgefundenen Munition kann nur in den seltensten Fällen angesehen werden, in welchem technischen Zustand sie sich befindet. Daher muss bei jedem Munitionsfund erst einmal von der Gefährlichkeit des Objektes ausgegangen werden, sodass entsprechende Sicherheitsmaßnahmen seitens der Forscher bzw. Bergungskräfte einzuleiten sind.

Eine weitere Beseitigungsmethode ist der Einsatz der Wasserstrahlschneidetechnik bei Sanierungen von subaquatischen „Kampfmittelaltlasten“ direkt am Fundort. Diese Technik kann auch durch unbemannte Tauchroboter erfolgen. ${ }^{19}$ In anderen Fällen können die gemeldeten Altlasten jedoch aufgrund ihrer Art und der geografischen Lage nur im Wege einer gezielten Sprengung beseitigt werden. Die Anwendbarkeit der Sprengung ist von den jeweils vorherrschenden Rahmenbedingungen im Einzelfall abhängig. Eine Minderung der Schallemission bei der Sprengung konventioneller Kampfmittel und eine Reduzierung der damit einhergehenden Gefährdung heimischer Meeressäuger sind durch den Einsatz von Blasenvorhängen möglich. ${ }^{20}$ Über die geeignete technische Ausstattung für die Beseitigung von Munitionsaltlasten werden im Regelfall die öffentlichen Behörden (Kampfmittelräumdienste) oder private Unternehmen verfügen. Jedoch könnte aufgrund der besonderen Lage zu Wasser die Bundesmarine eine geeignetere technische und personelle Ausstattung haben. ${ }^{21}$

\subsection{Umweltbelastungen infolge der Beseitigungsmaßnahmen}

Die Beseitigung der Munitionsaltlast durch kontrollierte Sprengungen stellt regelmäßig eine große Belastung für die Flora und Fauna des davon betroffenen Gewässer- gebiets dar. ${ }^{22}$ So sind die Nord- und die Ostsee unter anderem die Lebensräume von existenziell bedrohten Tierarten wie der Schweinswale (Phocoena phocoena), Seehunde (Phota vitulina) und Kegelrobben (Halichoerus grypus). Durch die mit der Sprengung verbundenen Druckwellen kann es zu lebensbedrohlichen Verletzungen oder gar Tötungen in einem Umkreis von mehreren Kilometern kommen. ${ }^{23}$ Die Detonationen bewirken, dass selbst bei einer weiten Distanz zu der jeweiligen Sprengung die Hörorgane der Meeressäugetiere dauerhaft geschädigt werden. Auch Lungenrisse, Blutungen im Hörorgan oder Gehirn können durch die Schallwelle einer Unterwasserexplosion hervorgerufen werden. ${ }^{24}$ Aber auch außerhalb des Wassers haben Sprengungen von Munitionsaltlasten negative Folgen für die Tierwelt. Die Seekälber werden ungefähr für fünf Wochen von den Mutterkühen an einem Strand oder in einer Küstenbucht gesäugt. In Folge einer Sprengung kann es dann zur Flucht der Muttertiere kommen. Zudem werden Riffe und die Pflanzenwelt (Plankton etc.) im Umkreis der Sprengung dauerhaft zerstört, was die Verschlechterung des ökologischen $\mathrm{Zu}$ standes des Gewässers zur Folge hat. Somit ist die Entscheidung für eine Munitionsaltlastensprengung als Gefahrenabwehrmaßnahme stets vor dem Hintergrund der gleichzeitigen Inkaufnahme von Meeresumweltbelastungen zu treffen.

\section{Rechtliche Implikation}

Im Zuge der Anordnung und Durchführung der Beseitigungsmaßnahmen stellen sich eine Reihe rechtlicher Fragen; allem voran von wem diese Maßnahmen durchzuführen sind und auf welcher Rechtsgrundlage sie erfolgen dürfen. Des Weiteren soll wegen der oftmals unzureichenden technischen und personellen Ausstattung der zivilen „Akteure“ der Frage nachgegangen werden, ob und inwieweit die Bundesmarine mit ihrem technischen und personellen „Knowhow“ bei der Beseitigung Unterstützung leisten darf. Schließlich werden dann mögliche materiell-rechtliche Beschränkungen einer Munitionsaltlastensprengung durch das Gewässerschutz- und Naturschutzrecht erörtert.

14) Greb, NordÖR 2011, 318.

15) Koske/Straumer, Wissenschaft erleben $2 / 2018,10,11$.

16) Koske/Straumer, Wissenschaft erleben $2 / 2018,10$.

17) Koske/Straumer, Wissenschaft erleben $2 / 2018,10,11$

18) Wichert, Marine Forum (Bd. 5) 2012, S. 25-27.

19) BT-Drs. 19/23573, S. 2; Forschungszentrum Jülich GmbH: RoBEMM - „Robotisches Unterwasser-Bergungs- und Entsorgungsverfahren inkl. Technik zur Delaboration von Munition im Meer", Stand 21.9.2021, abrufbar unter https://www ptj.de/lw_resource/datapool/systemfiles/agent/ptjpublications/ 7D4DE0AED845727DE0539A695E8694E1/live/document/ Tagungsband_MGS_2018_red.pdf, S. $149 \mathrm{ff}$.

20) Dazu näher unten 2.3.1.2

21) So sprengten Marineeinheiten im Rahmen eines Übungsmanövers Minen in der Ostsee, vgl. hierzu: Der Spiegel: Bundeswehr 18 Schweinswale nach Minensprengung in der Ostsee tot, Stand 21.9.2021, abrufbar unter https://www.bundeswehr.de/de/organisation/marine/aktuelles/northern-coasts-2019-deu-marfor.

22) Helmholtz Perspektiven: Das Erbe der Kriege, Stand 21.9.2021, abrufbar unter https://www.helmholtz.de/fileadmin/user_upload/04_mediathek/perspektiven/epaper-Perspektive__03 2018/index.html\#32, S. 33, 35.

23) Tagesschau: Verendete Schweinswale Tod durch Explosionstrauma, Stand 21.9.2020, abrufbar unter https://www.tagesschau.de/inland/schweinswale-105.html.

24) Bundesamt für Naturschutz (BfN): Auswirkungen der Sprengungen von Seeminen im Naturschutzgebiet „Fehmarnbelt“ Ende August 2019, Stand 21.9.2021, abrufbar unter https:// www.bfn.de/fileadmin/BfN/meeresundkuestenschutz/Dokumente/Minensprengungen_im_Fehmarnbelt/Gesamtbewertung_Fehmarnbelt_Minensprengungen.pdf, S. 3. 


\subsection{Zuständigkeitsfragen}

Zunächst ist voranzuschicken, dass es angesichts fehlender spezialgesetzlicher Regelungen für den Umgang mit Munitionsaltlasten dabei lediglich um Maßnahmen der behördlichen Gefahrenabwehr gehen kann. ${ }^{25}$ Wobei der Gefahrenbegriff auf See demjenigen des materiellen Polizeirechts entspricht. Demgemäß umfasst Gefahrenabwehr alle Aufgabenbereiche, die im Zusammenhang mit der Abwehr von Gefahren für die öffentliche Sicherheit und Ordnung stehen. ${ }^{26}$

Dabei ist zunächst von Bedeutung, ob die Zuständigkeit für die Beseitigungsmaßnahmen beim Bund oder dem jeweiligen Bundesland bzw. bei den Bundes- oder Landesbehörden liegt. Dies hängt maßgeblich von dem jeweiligen Ort ab, an dem sich die Munitionsaltlast befindet. So ist für die Kompetenzzuordnung nicht zuletzt entscheidend, ob sich die Munition innerhalb der deutschen Hoheitsgrenzen befindet oder nicht.

Die Grundlage für eine entsprechende Zuordnung bildet insoweit das UN-Seerechtsübereinkommens von 1982 $(\mathrm{SRU})^{27}$, das auf der völkerrechtlichen Ebene zwischen verschiedenen räumlichen Kompetenzzonen unterscheidet. Danach sind für die hier relevanten spezifisch „deutschen“ Verhältnisse als zu unterscheidende Meereszonen in erster Linie das Küstenmeer und die ausschließliche Wirtschaftszone (AWZ) bedeutsam. ${ }^{28}$ Hierbei entspricht dem völkerrechtlich verwendeten Begriff des Küstenmeeres derjenige des auf das deutsche Hoheitsgebiet bezogenen Küstengewässers im einschlägigen deutschen Gewässerschutzrecht (vgl. $₫ 2$ Abs. 1 S. 1 Nr. 2, $\$ 3$ Nr. 2 WHG) ${ }^{29}$ Demgegenüber erstrecken sich die in $\$ 2$ Abs. $1 \mathrm{a}, \$ 3 \mathrm{Nr}$. $2 \mathrm{a} W \mathrm{WG}$ zugrundegelegten Meeresgewässer begrifflich darüber hinaus auch auf die AWZ und den deutschen Festlandssockel.

\subsubsection{Innerstaatliche Zuständigkeiten}

Die Bundesrepublik hat mit der Proklamation vom 11.11.1994 in Übereinstimmung mit dem SRÜ die Breite des Küstenmeeres in der Nordsee auf 12 Seemeilen (= 22,224 Kilometer) festgelegt. ${ }^{30}$ In der Ostsee ist die Festlegung auf ein entsprechend breites Küstenmeer für die Anliegerstaaten aus topografischen Gründen nicht möglich; hier wird die seewärtige Grenze des Küstenmeeres durch geografische Koordinaten festgelegt, die zum Teil deutlich weniger als 12 Seemeilen von der Küstenlinie bzw. der Basislinie entfernt sind.

Befindet sich die Altlast innerhalb dieser 12-Seemeilenzone, also im Bereich des Küstengewässers, ist zu klären, ob dem Bund oder den jeweiligen Ländern die Zuständigkeit bezüglich der Gefahrenabwehr von Munitionsaltlasten obliegt. Dies bestimmt sich grundsätzlich nach den Artt. 30 und 83 ff. GG. Demnach gilt, dass die Verteilung der Verwaltungskompetenzen zwischen Bund und Ländern ausschließlich ist, d.h. entweder der Bund oder die Länder zuständig sind. ${ }^{31}$ Gemäß Art. 30 GG obliegt die Ausübung der staatlichen Befugnisse und die Erfüllung der staatlichen Aufgaben den Ländern, soweit das Grundgesetz keine anderen Regelungen trifft oder zulässt. Letzteres ist mit den Artt. 86 ff. GG geschehen, die für die dort bestimmten Fälle eine bundeseigene Verwaltung vorsehen. Deswegen sollen zunächst deren hier möglicherweise sachlich einschlägigen Anwendungsbereiche ermittelt werden, um sodann die den Ländern verbleibenden Vollzugskompetenzen feststellen zu können.

\subsubsection{Bundeseigenverwaltung}

Eine obligatorische Bundesverwaltung sieht Art. 87 Abs. 1 S. 1 GG für die Verwaltung der Bundeswasserstraßen und der Schifffahrt vor. ${ }^{32}$ Eine Verbandskompetenz des Bundes könnte sich demnach aus der Wasserstraßenverwaltung (Art. 89 Abs. 2 S. 1 GG) oder aus der Seeschifffahrtsverwaltung (Art. 89 Abs. 2 S. 2 GG) ergeben:
Die Munitionsaltlastensprengung innerhalb des Küstengewässers fällt gem. Art. 89 Abs. 2 S. 1 GG grundsätzlich unter die administrative Zuständigkeit der Bundesverwaltung, soweit es sich bei dem Küstengewässer um eine Bundeswasserstraße handelt. $\ 1$ des Bundeswasserstraßengesetzes (WaStrG) ${ }^{33}$ unterscheidet die Bundeswasserstraßen in Binnenwasserstraßen ( $\$ 1$ Abs. 1 Nr. 1 WaStrG) und Seewasserstraßen ( $\$ 1$ Abs. 1 Nr. 2 WaStrG). So sind Seewasserstraßen die Flächen zwischen der Küstenlinie bei mittlerem Hochwasser oder der seewärtigen Begrenzung der Binnenwasserstraßen und der seewärtigen Begrenzung des Küstengewässers. Davon umfasst sind nicht nur die dem allgemeinen Verkehr dienenden Teile der Küstengewässer wie etwa die Fahrrinnen, sondern sämtliche mit Wasser bedeckten Teile. Demnach erstreckt sich die Bundeswasserstraße und deren bundeseigene Verwaltung räumlich auf das gesamte Küstenvorfeld bis hin zur deutschen Hoheitsgrenze. Doch bezieht sich der sachliche Anwendungsbereich der Wasserstraßenverwaltung nicht auf alle denkbaren Aspekte im Zusammenhang mit Bundeswasserstraßen, sondern beschränkt sich lediglich auf deren Funktion als Verkehrsweg. ${ }^{34}$ In diesem Zusammenhang werden Maßnahmen zur Ausführung des Wasserstraßengesetzes, insbesondere der Neu- und Ausbau und die Unterhaltung von Bundeswasserstraßen einschließlich ihrer Planung sowie die Strompolizei erfasst. ${ }^{35}$ In Betracht kommt hier aber allenfalls ein gefahrenabwehrrechtlicher Bezug zur Strompolizei. Gemäß $\$ 24$ Abs. 1 WaStrG haben die Behörden der WasserstraBen- und Schifffahrtsverwaltung des Bundes die Aufgabe, zur Gefahrenabwehr Maßnahmen zu treffen, die nötig sind, um die Bundeswasserstraßen in einem für die Schifffahrt erforderlichen Zustand zu erhalten. So ist die Strompolizei als Wegepolizei abzugrenzen von der noch zu erörternden Schifffahrtspolizei, welche eine reine Verkehrspolizei darstellt ( $\mathbb{1}$ Abs. 1 Nr. 2 BinSchAufgG, $\$ 1$ Nr. 2 SeeaufgG). ${ }^{36}$ $\mathrm{Zu}$ dem für die Schifffahrt erforderlichen Zustand der Bundeswasserstraße gehört wegen ihrer Verkehrsfunktion, insbesondere die Freihaltung von Schifffahrtshindernissen. ${ }^{37}$ Eine Verbesserung des für die Schifffahrt erforderlichen Zustandes ist hingegen nicht die Aufgabe der Strompolizei. $^{38}$ Die strompolizeiliche Beseitigungsmaßnahme der Behörden der Wasserstraßen- und Schifffahrtsverwaltung des Bundes stellt somit eine Maßnahme ,zur Gefahrenabwehr" i.S.d. $\$ \$ 24$ Abs. 1, 28 WaStrG dar. Daraus folgt,

25) So zutreffend auch Jenisch, NordOer, 493, 494

26) BVerfGE, Beschl. v. 14. 5. 1985 - 1 BvR 233/81, 1 BvR 341/81, NJW 1985, 2395, 2398; BVerwG, Urt. v. 28.3.2012 - 6 C 12/11, NJW 2012, $2676 \mathrm{f}$.

27) Seerechtsübereinkommen der Vereinten Nationen v. 10.12.1982, BGB1. 1994 II S. 1798.

28) Jenisch, NuR 2006, 79, 80

29) So wohl auch Mohr, NordOer 2020, 389, 390.

30) Proklamation der Bundesregierung über die Ausweitung des deutschen Küstenmeeres v. 11.11. 1994, BGB1. 1994 I S. 3428.

31) Hellermann, in: Epping/Hillgruber, BeckOK GG, 7. Ed. 15. 5.2021, Art. 30 Rdnr. 22.

32) Zum schlichten Verweisungscharakter des Art. 87 Abs. 1 S. 1 GG, siehe Suerbaum, in: Epping/Hillgruber, BeckOK GG, 47 Ed. 15. 5.2021, Art. 87 Rdnr. 19.

33) Bundeswasserstraßengesetz (WaStrG) v. 23.5.2007, BGBl. I S. 962; 2008 I S. 1980; zuletzt geändert durch Art. 57 des Gesetzes v. 23.6.2021, BGBl. I S. 1858

34) Remmert, in: Epping/Hillgruber, BeckOK GG, 47. Ed. 15. 5. 2021 , Art. 89 Rdnr. 9.

35) Remmert, in: Epping/Hillgruber, BeckOK GG, 47. Ed. 15. 5.2021, Art. 89 Rdnr. 9.

36) Reinhardt/Schäfer, in: dies., WaStrG, 3. Online-Auflage 2017, $\$ 24$ Rdnr. 1.

37) OVG Frankfurt (Oder), Beschl. v. 1.12.1999 - 4 B 103/99, BeckRS 2000, 20206 Rdnr. 5.

38) Reinhardt/Schäfer, in: dies., WaStrG, 3. Online-Auflage 2017, $₫ 24$ Rdnr. 2. 
dass eine Gefahrenlage oder die bereits eingetretene Störung erforderlich ist, wohingegen eine latente Gefahr nicht ausreicht. ${ }^{39}$ Eine Gefahrenlage liegt bereits vor, wenn die erkennbare Möglichkeit, nicht dagegen die unbedingte Gewissheit besteht, dass der Verkehrsablauf durch eine Munitionsaltlast beeinträchtigt oder gefährdet wird. Ausreichend ist, wenn sich die Munitionsaltlast - jedenfalls teilweise in der Fahrrinne einer Bundeswasserstraße befindet. ${ }^{40}$ Eine Beseitigungsmaßnahme durch die Bundeswasserstraßenverwaltung ist im Ergebnis also nur insofern rechtlich zulässig und geboten, als sich die Munitionsaltlasten innerhalb der Bundeswasserstraßen befinden und die Sicherheit des Schiffsverkehrs beeinträchtigen.

Als fakultative Bundesverwaltung kann sich die administrative Zuständigkeit zur Munitionsaltlastenbeseitigung aber auch aus der Bundesschifffahrtsverwaltung (Art. 89 Abs. 2. S. 2 GG) ergeben. Notwendig ist jedoch im Gegensatz zu Art. 89 Abs. 2 S. 1 GG, dass der Bund eine Befugnis auf Grund eines einfachen Bundesgesetzes haben muss. Diese hat er u.a. durch das Seeaufgabengesetz (SeeAufG) ${ }^{41}$. Demnach obliegt dem Bund gemäß $\$ 1$ Nr. 2 SeeAufG die Abwehr von Gefahren für die Sicherheit und Leichtigkeit des Verkehrs sowie die Verhütung von der Seeschifffahrt ausgehenden Gefahren und schädlichen Umwelteinwirkungen i.S.d. Bundes-Immissionsschutzgesetzes (Schifffahrtspolizei) auf den Seewasserstraßen. In jedem der Fälle geht es dort aber lediglich um die Abwehr seeverkehrsspezifischer Gefahren, die den normalen Verkehrsablauf behindern. ${ }^{42}$ Die Abwehr von äußeren, nichtbetriebsspezifischen Gefahren gehört indes nicht dazu. ${ }^{43}$ Nur falls Munitionsaltlasten tatsächlich den normalen Verkehrsablauf bedrohen, liegt die Beseitigungspflicht auch insoweit beim Bund ${ }^{44}$ und dessen Behörden der Wasserstraßen- und Schifffahrtsverwaltung. Diese haben ausschließlich dann nach pflichtgemäßem Ermessen die notwendigen Maßnahmen zu treffen (vgl. \$3 Abs. 1 SeeAufgG).

\subsubsection{Landeseigenverwaltung}

$\mathrm{Zu}$ den Bundesländern mit Küstenbezug gehören Bremen, Hamburg, Mecklenburg-Vorpommern, Niedersachsen und Schleswig-Holstein. Deren Landesvollzugskompetenzen i.S. d. Art. 30 GG zur Beseitigung der Munitionsaltlasten könnten sich allem voran wegen der negativen Einwirkungen der Munitionsaltlasten auf das Medium Wasser aus dem vom Bund erlassenen Wasserhaushaltsgesetz (WHG) ergeben, das die jeweiligen Länder aufgrund der Artt. $83 \mathrm{f}$. GG als eigene zu vollziehen haben.

Gemäß $\$ 1$ WHG zielt dieses Gesetz darauf, die Gewässer als Bestandteil des Naturhaushalts, als Lebensgrundlage des Menschen, als Lebensraum für Tiere und Pflanzen sowie als nutzbares Gut durch eine nachhaltige Gewässerbewirtschaftung zu schützen. Unter den Gewässerbegriff fällt gem. $\$ 2$ Abs. 1 S. 1 Nr. 2 i. V.m. $₫ 3$ Nr. 2 WHG auch das Küstengewässer, ${ }^{45}$ wodurch der räumliche Anwendungsbereich des Gesetzes eröffnet ist. Der Schutzzweck ist dabei nicht nur im Sinne der konkreten Gefahrenabwehr zu verstehen, sondern umfasst auch die Gefahrenvorsorge. ${ }^{46}$ Mit \$6 Abs. 1 Nr. 1, 2 und 7 WHG hat der Gesetzgeber verdeutlicht, dass dem Staat eine ökologische Schutzverantwortung hinsichtlich der Qualität und Quantität des Gewässers obliegt. ${ }^{47}$ Demnach muss er dafür Sorge tragen, dass trotz der Unvermeidbarkeit einer direkten oder indirekten Beeinflussung durch den Menschen die Ökofunktionalität der jeweiligen Gewässer erhalten bleibt. ${ }^{48}$ Um diese Schutzverantwortung gewährleisten zu können, hat der Bundesgesetzgeber den Wasseraufsichtsbehörden u.a. die wasserpolizeiliche Generalklausel gemäß \100 Abs. 1 S. 2 WHG an die Hand gegeben. ${ }^{49}$ Danach ordnet die zuständige Behörde nach pflichtgemäßem Ermessen insbesondere solche Maßnahmen an, die im Einzelfall notwendig sind, um Beeinträchtigungen des Wasserhaushalts zu vermeiden oder zu beseitigen. Der sachliche Anwendungsbereich des
WHG ist eröffnet, sofern die Munitionsaltlast eine Beeinträchtigung des Wasserhaushalts darstellt; was etwa der Fall ist, wenn das Vorhandensein der Altlasten eine erheblich nachteilige Auswirkung für die ökologische Funktionalität des betroffenen Gewässers zur Folge hätte. ${ }^{50}$ Es ist davon auszugehen, dass Munitionsaltlasten, die aufgrund von Alterungsprozessen der Korrosion erlegen sind, toxische Inhaltsstoffe freisetzen, die negative Auswirkungen auf das Wasser und die Lebewesen im Küstengewässer haben können. ${ }^{51}$ Insoweit zumindest ergibt sich eine Zuständigkeit der nach Landesrecht zuständigen Wasserbehörden, denen darauf bezogen die Wahrnehmung wasserpolizeilicher Aufgaben obliegt..$^{52}$

Demgegenüber ist der den Ländern überantwortete $\mathrm{Ka}$ tastrophenschutz Teil der allgemeinen Gefahrenabwehr. So gesehen könnten die Katastrophenschutzgesetze der jeweiligen Küstenländer entsprechende Maßnahmeermächtigungen für die Landesbehörden begründen. ${ }^{53}$ Die zentrale Aufgabe des Katastrophenschutzes ist indes der Schutz vor und das Management von Katastrophenfolgen. Die Katastrophenschutzgesetze betreffen üblicherweise sowohl die Bevölkerung als auch die hilfeleistenden Organe und Hilfskräfte und schließlich auch die Behörden selbst. Doch wird es für ein behördliches Einschreiten insoweit regelmäßig bereits am Vorliegen einer durch einen entsprechend großen Schadensfall geprägten Katastrophenlage fehlen. ${ }^{54}$ Denn allein die von den vorhandenen Munitionsaltlasten ausgehende Gefährlichkeit begründet jedenfalls schon keinen Schadensfall.

Die allgemeinpolizeiliche Gefahrenabwehr liegt aufgrund der verfassungsmäßigen Kompetenzverteilung zwischen Bund und Ländern bei letzteren und deren Behörden. ${ }^{55}$ Räumlich sind diese freilich jeweils auf die Ausübung innerhalb der jeweiligen Länder-Hoheitsgewässer beschränkt. Anzuwendende Regelungen befinden sich in den jeweiligen allgemeinen Polizei- bzw. Ordnungsgesetzen der Küstenländer. ${ }^{56}$ Danach gehört es zu den Aufgaben der allgemeinpolizeilichen Gefahrenabwehr auf See, allgemeine Straftaten und Ordnungswidrigkeiten sowie die

39) So zutreffend auch Jenisch, NordÖR 2020, 493, $494 \mathrm{f}$.

40) OVG Frankfurt (Oder), Beschl. v. 1.12.1999 - 4 B 103/99, BeckRS 2000, 20206 Rdnr. 10.

41) Seeaufgabengesetz in der Fassung der Bekanntmachung v. 17.6.2016, BGBl. I S. 1489; zuletzt geändert durch Art. 4 der Verordnung vom 3.3.2020, BGB1. I S. 412.

42) Jenisch, NordÖR 2020, 493, 494.

43) Ehlers, SeeAufG, 4. Aufl. 2019, \$1 Rdnr. 16

44) So zutreffend auch Jenisch, NordÖR 2020, 493, 494.

45) Vgl. Kotulla, WHG, 2. Aufl. 2011, § 3 Rdnr. $19 \mathrm{ff}$.

46) Statt aller Kotulla, WHG, 2. Aufl. 2011, $₫ 1$ Rdnr. 5.

47) Kotulla, WHG, 2. Aufl. 2011, §6 Rdnr. 8

48) Kotulla, WHG, 2. Aufl. 2011, \$6 Rdnr. 8.

49) Kotulla, WHG, 2. Aufl. 2011, \$100 Rdnr. 18

50) Kotulla, WHG, 2. Aufl. 2011, \$100 Rdnr. 19.

51) Siehe: 1.1 Gefahrenpotential für Menschen und Meeresumwelt.

52) Wahlen, Maritime Sicherheit im Bundesstaat kompetenzrechtliche Herausforderungen auf dem Weg zu einer Deutschen Küstenwache, 2012, S. 88, 101.

53) Für die genannten Küstenländer: Bremen (BremHilfeG - Brem. GB1. 2002, Nr. 25), Hamburg (HmbKatSG - HmbGVB1. I978, S. 31), Mecklenburg-Vorpommern (LKatSG M-V - GVOB1. M-V 2016, S. 611, 793), Niedersachsen (NKatSG - Nds. GVB1. 2002, S. 73), Schleswig-Holstein (LKatSG - GVOBl. 2000, S. 664).

54) $\mathrm{Zu}$ dem sachlichen Anwendungsbereichs des Katastrophenrecht siehe: Gusy, GSZ 2020, $101 \mathrm{ff}$.

55) Hellermann, in: Epping/Hillgruber, BeckOK GG, 7. Ed 15. 5.2021, Art. 30 Rdnr. 1-6; Jenisch, NordOer 2020, 493, $494 \mathrm{f}$

56) Für die genannten Küstenländer: Bremen (BremPolG - Brem. GB1. 2001, 441), Hamburg (SOG HH - HmbGVB1. I966, S. 77), Mecklenburg-Vorpommern (SOG MV - GVOB1. M-V. 2011, S. 246), Niedersachsen (Nds.SOG - Nds. GVB1. 2005, S. 9), Schleswig-Holstein (LVwG-SH - GVOBl. 1992, 243, 534). 
Abwehr von Gefahren für Individualrechtsgüter wie Leib oder Leben $\mathrm{zu}$ verhüten. Innerhalb der Landesbehörden sind für die Gefahrenabwehr zur See besonders die Wasserschutzpolizeien zur allgemeinpolizeilichen und die Kampfmittelräumdienste zur besonderen polizeilichen Gefahrenabwehr innerhalb der Hoheitsgewässer des Landes berufen.

2.1.2 Vollzugszuständigkeiten außerhalb der Hoheitsgrenzen (AWZ)

Bleibt noch zu klären, wem die administrative Zuständigkeit für die Beseitigung von Munitionsaltlasten im Gewässerbereich außerhalb der deutschen Hoheitsgrenzen, also in der AWZ, zufällt. Die AWZ ist gemäß Art. 55, 57 SRÜ ein jenseits des Küstenmeeres gelegenes Gebiet, dessen Bereich sich - zumindest in der Nordsee - bis zu 200 Seemeilen (sm) ab der Basislinie abzüglich der maximalen Küstenbreite von $12 \mathrm{sm}$ erstreckt, wobei die Ausdehnung auf ein entsprechend breites Küstenmeer in der Ostsee, für die Bundesrepublik aus topografischen Gründen nicht in Betracht kommt. Als Nichtbestandteil des Hoheitsgebietes der Bundesrepublik unterliegt die AWZ gemäß Teil V der SRÜ einer besonderen Rechtsordnung. ${ }^{57}$ Das deutsche Recht erlangt dort grundsätzlich keine Geltung. ${ }^{58}$ Etwas anderes gilt nur, wenn die Anwendung deutscher Rechtsvorschriften in der AWZ ausdrücklich gesetzlich statuiert ist. Dies ist etwa mit $₫ 1$ Nr. 3 lit. b) SeeAufgG geschehen. Danach betreffen die polizeilichen Befugnisse des Bundes in der AWZ über die Schifffahrtspolizei hinaus auch die Abwehr von Gefahren und die Beseitigung von Störungen der öffentlichen Sicherheit oder Ordnung in sonstigen Fällen. Dabei reicht es aus, dass ein unmittelbarer Seeschifffahrtsbezug besteht. ${ }^{59}$ Gleichwohl geht es in der AWZ neben der Abwehr lediglich seeverkehrsspezifischer Gefahren ${ }^{60}$, lediglich um Fälle wie der Abfallbeseitigung auf See, des Drogenhandels, des Schmuggels, der Sklaventransporte, unerlaubte Rundfunksendungen, Beschädigungen von Telegraphenkabeln oder des Gesundheitsschutzes der Seeleute. ${ }^{61}$ Gemäß $\$ 3$ Abs. 2 SeeAufgG haben in solchen Fällen die Bundesbehörden der Wasser- und Schifffahrtsverwaltung die notwendigen Handlungen zur Abwehr der Gefahren nach pflichtgemäBen Ermessen zu treffen.

\subsection{Amtshilfe durch die Bundeswehr}

Da die zivilen Vollzugsbehörden oft nur über eine unzureichende personelle wie sachliche Ausstattung für die Beseitigung von Munitionsaltlasten verfügen, die die Wahrnehmung einer effektiven Gefahrenabwehr mitunter einschränkt oder gar unmöglich macht, stellt sich die Frage, ob und inwiefern die Bundeswehr bei der Beseitigung von Munitionsaltlasten unterstützend tätig werden darf. Denn diese kann in der Regel auf gut geschultes Personal sowie eine besser ausgestattete Kampfmittelaufsuchungs- und -beseitigungsausrüstung zugreifen. Als Rechtsgrundlage für eine derartige Unterstützung kommt Art. 35 Abs. 1 GG in Betracht. Danach haben sich alle Behörden des Bundes und der Länder gegenseitig Amts- und Rechtshilfe leisten. Auf diese Weise können in geeigneten Fällen nicht nur Verwaltungsabläufe im Rahmen der bundesstaatlichen Ordnung vereinfacht, sondern es kann auch eine Steigerung der Verwaltungseffektivität erreicht werden. ${ }^{62}$ Anerkanntermaßen gehört die Bundeswehr zu den grundsätzlich amtshilfefähigen Behörden. ${ }^{63}$ Dennoch bleibt zu klären, ob eine Amtshilfe der Bundeswehr mit Blick auf den hier interessierenden Fall der Munitionsbeseitigung statthaft wäre.

\subsubsection{Behördeneigenschaft}

Die Bundeswehr darf nämlich nur in dem ihr von Art. 35 Abs. 2 und 3 GG zugebilligten Rahmen Hilfe leisten. Nicht von der Amtshilfekompetenz der Bundeswehr gedeckt sind die Aufgaben i. S. d. Art. 87a Abs. 2 GG. ${ }^{64}$ Der Rechtsprechung des BVerfG zufolge muss bei der Hilfe durch die
Bundeswehr zwischen einer spezifisch militärischen Nutzung der Streitkräfte mit ihrem Droh- und Einschüchterungspotential und einer bloß technisch-unterstützenden Hilfeleistung unterschieden werden. ${ }^{65}$ So sind etwa das Schleppen von Sandsäcken zur Verstärkung von Deichen, die Rettung von Menschen mit Schlauchbooten und Hubschraubern aus überschwemmten Gebieten sowie Erntehilfen nicht als Einsatz i.S.d. Art. 87a Abs. 2 GG zu qualifizieren und deshalb von der Amtshilfe gemäß Art. 35 GG gedeckt. ${ }^{66} \mathrm{Da}$ sich die Beseitigung von Munitionsaltlasten im Wasser ausschließlich als eine bloß technisch-unterstützende Hilfeleistung darstellt, ist auch die Bundeswehr insoweit amtshilfefähig.

\subsubsection{Tatbestandsvoraussetzungen} und Umfang der Amtshilfe

Zunächst müsste seitens der zuständigen Vollzugsbehörde ein Hilfsersuchen an die Bundeswehr gerichtet werden. ${ }^{67}$ Denn nur derjenigen Behörde kann Hilfe geleistet werden, die Hilfe benötigt. ${ }^{68} \mathrm{Um}$ eine aufgedrängte Amtshilfe $\mathrm{zu}$ vermeiden, hat die Behörde in ihrem Aufgabenbereich eigenverantwortlich zu prüfen, ob und inwieweit Hilfe erforderlich ist, fremde Unterstützungsleistungen für zulässig und zweckmäßig erachtet und von welcher anderen Behörde sie Hilfe anfordern will. ${ }^{69}$ Führt die Bundeswehr die Sprengung der Munitionsaltlast durch, ohne dass die Vollzugsbehörde mittels eines Hilfeersuchens darum gebeten hat, ist diese Maßnahme jedenfalls nicht mehr durch die Amtshilfe nach Art. 35 GG gedeckt. Dann könnte es sich allenfalls um eine „Spontanhilfe“"70 in Form der öffentlichrechtlichen Geschäftsführung ohne Auftrag handeln. Dagegen ist jedoch einzuwenden, dass durch die Anerkennung der öffentlich-rechtlichen GoA die staatliche Kompetenzordnung unter Rückgriff auf ein zivilrechtliches Institut geradezu generalklauselartig unterlaufen wird. ${ }^{71}$ Ferner müsste die Vollzugsbehörde für eine Amtshilfe das Ersuchen hinreichend bestimmt haben, die Bundeswehr zweifelsfrei über die konkret vorzunehmende Beseitigung der Munitionsaltlast informieren ${ }^{72}$ und die von der Bundeswehr angeforderte Hilfe aus tatsächlichen Gründen nicht selbst vornehmen können. ${ }^{73}$ Zudem darf die Vollzugsbe-

57) Wolf, NuR 2005, 375, 378.

58) Wolf, NuR 2005, 375, 378.

59) BVerwG, Urt. v. 14.7.2011 - 7 C 7.10, Rdnr. 10

60) So auch Jenisch, NordÖR 2020, 493, 495.

61) Ehlers, SeeAufG, 4. Aufl. 2019, \1 Rdnr. 25

62) Bauer, in: Dreier, Grundgesetz-Kommentar, 3. Aufl. 2015 , Art. 35 Rdnr. 10.

63) Epping, in: ders./Hillgruber, BeckOK GG, 47. Ed. 15.5.2021, Art. 35 Rdnr. 2.

64) Epping, in: ders./Hillgruber, BeckOK GG, 47. Ed. 15.5.2021, Art. 35 Rdnr. 2.

65) BVerfG, Beschl. v. 3.7. 2012 - 2 PBvU 1/11, NVwZ 2012 , Rdnr. 1239, 1244; BVerfGE, Beschl. v. 20.3.2013 - 2 BvF 1/05, NVwZ 2013, Rdnr. 242, 269.

66) Epping, in: ders./Hillgruber, BeckOK GG, 47. Ed. 15.5.2021, Art. 35 Rdnr. 2.

67) BGH, Urt. v. 16.1.1961 - III ZR 210/59, NJW 1961, $918 \mathrm{f}$; Bauer, in: Dreier, Grundgesetz-Kommentar, 3. Aufl. 2015 , Art. 35 Rdnr. 19.

68) Wahlen, Maritime Sicherheit im Bundesstaat kompetenzrechtliche Herausforderungen auf dem Weg zu einer Deutschen Küstenwache, S. 151

69) Dederer, in: Maunz/Dürig, Grundgesetz-Kommentar, 94. EL Januar 2021, Art. 35 Rdnr. 45.

70) Zum Streit um die Verzichtbarkeit eines Ersuchens der zuständigen Behörde (Spontanhilfe) vgl. Dederer, in: Maunz/Dürig, Grundgesetz-Kommentar, 94. EL Januar 2021, Art. 35 Rdnr. 46 m.w. N.

71) Schoch, Die Verwaltung (38) 2005, 91, $98 \mathrm{f}$.

72) Danwitz, in: v. Mangoldt/Klein/Starck, Grundgesetz, 7. Aufl. 2018, Art. 35 Rdnr. 19.

73) Meyer-Teschendorf, JuS 1981, 187, 190. 
hörde ihre Befugnisse nicht mittels der Amtshilfevorschriften erweitern ${ }^{74}$ und es muss feststehen, dass die Bundeswehr rechtlich und tatsächlich in der Lage ist, die nachgesuchte Beseitigung durchführen zu können. ${ }^{75}$

Die Amtshilfe durch die Bundeswehr umfasst sämtliche Unterstützungstätigkeiten von der Erteilung von Auskünften über die Zurverfügungstellung von Mitteln bis hin zur Vornahme von Vollzughandlungen in Form der eigentlichen Munitionsbeseitigung. Nimmt die Bundeswehr die Beseitigung selbst vor, handelt sie dabei nach dem für sie geltenden eigenen Recht und unterliegt nicht den Weisungen der hilfesuchenden Behörde.

\subsection{Verwaltungsrechtlicher Rahmen}

Die mit den Sprengungen der Munitionsaltlasten verbundenen negativen Folgen für die Meeresflora und -fauna sowie für die Qualität des (Meer-)Wassers könnten jedoch insbesondere den Bestimmungen des Wasserhaushaltsgesetzes $(\mathrm{WHG})^{76}$ und des Bundesnaturschutzgesetzes (BNatSchG $)^{77}$ zuwiderlaufen; was die Rechtswidrigkeit dieser Beseitigungsmaßnahmen zur Folge hätte.

\subsubsection{Erlaubnispflichtige Benutzung}

i. S.d. $\iint 8$ Abs. 1, 9 Abs. 2 Nr. 2 WHG

Bei einer Altlastensprengung wird es sich regelmäßig um eine nach $₫ 8$ Abs. 1 WHG erlaubnispflichtige Benutzung i. S.d. $\$ 9$ Abs. 2 Nr. 2 WHG handeln, da die Sprengung geeignet ist, dauernd oder in einem nicht nur unerheblichen Ausmaß nachteilige Veränderungen der Wasserbeschaffenheit - gemeint ist hier die physikalische, chemische oder biologische Beschaffenheit des Wassers eines Küstengewässers (vgl. \3 Nr. 9 WHG) - herbeizuführen. Etwas anderes gilt nach $\$ 8$ Abs. $2 \mathrm{~S}$. 1 WHG nur, wenn die Sprengung zur Abwehr einer nicht nur abstrakten, sondern gegenwärtigen Gefahr erfolgt. ${ }^{78}$ Nur dann darf sie erlaubnisfrei erfolgen.

Die Erteilung einer Erlaubnis kommt indes überhaupt nur in Betracht, sofern von der Sprengung keine schädlichen, zumindest aber durch Nebenbestimmungen vermeidbare oder ausgleichbare Gewässerveränderungen zu erwarten sind und ggf. nach anderen öffentlich-rechtlichen Vorschriften existierende Anforderungen erfüllt werden ( $\$ 12$ Abs. 1 WHG). Mit den schädlichen Gewässerveränderungen sind solche Veränderungen der Gewässereigenschaften gemeint, die das Wohl der Allgemeinheit, insbesondere die öffentliche Wasserversorgung, beeinträchtigen oder die nicht den Anforderungen entsprechen, die sich aus dem WHG, aus aufgrund des WHG erlassenen oder aus sonstigen wasserrechtlichen Vorschriften ergeben ( $\$ 9$ Nr. 10 WHG). Abgesehen von der hier eher unproblematischen Allgemeinwohlfrage wäre bei der Durchführung der Sprengungen aber an die zwingende Einhaltung bestimmter wasserrechtlicher Vorgaben zu denken.

\subsubsection{Bewirtschaftungsziele für das Küstengewässer ( $\int 44$ i. V.m. $\$ 27$ Abs. 1 WHG)}

So könnten einer Altlastensprengung vornehmlich die für die Küstengewässer gem. \44 i. V.m. \27 Abs. 1 WHG geltenden Bewirtschaftungsziele entgegenstehen. Demnach sind die Küstengewässer so zu bewirtschaften, dass eine Verschlechterung ihres ökologischen und ihres chemischen Zustands vermieden (Verschlechterungsverbot) ${ }^{79}$ sowie ein guter ökologischer und chemischer Zustand erhalten oder erreicht wird (Erhaltungs- und Verbesserungsgebot) ${ }^{80}$.

Eine Munitionsaltlastensprengung im Bereich des Küstengewässers darf somit nicht gegen das im $₫ 27$ Abs. 1 Nr. 1 WHG verankerte Verschlechterungsverbot verstoBen. Unter Verschlechterung sind alle nachteiligen Veränderungen des zurzeit bestehenden chemischen und/oder ökologischen Gewässerzustands zu verstehen. ${ }^{81}$ Unter dem „ökologischer Zustand“ ist nach Art. 2 Nr. 21 WRRL „die Qualität von Struktur und Funktionsfähigkeit aquatischer, in Verbindung mit Oberflächengewässern stehenden Ökosysteme gemäß der Einstufung nach Anh. V" zur WRRL zu verstehen. Die Bewertung des jeweiligen Gewässerzustandes richtet sich nach den Vorgaben in Nr. 1.2 Anh. V WRRL, dem im deutschen Recht insoweit die Tabelle 5 der Anlage 4 zur Oberflächengewässerverordnung ${ }^{82}$ entspricht. Der chemische Zustand beschreibt hingegen die qualitativen Eigenschaften des Wassers unter Bezugnahme auf die einschlägigen gemeinschaftsrechtlichen Schadstoffgrenzwerte. ${ }^{83}$ So liegt ein ,guter chemischer Zustand eines Oberflächengewässers" nach Art. 2 Nr. 24 WRRL vor, wenn kein Schadstoff in einer über die Vorgaben des Anh. IX, der nach Art. 16 Abs. 7 ergangenen oder sonstigen einschlägigen Umweltqualitätsnormen hinausgehenden Konzentration vorliegt. ${ }^{84}$ Sollte es durch eine Sprengung zur Verschlechterung des ökologischen oder chemischen Zustandes in dem jeweiligen Küstengewässer kommen, könnte eine etwaige Abweichung von den Bewirtschaftungszielen jedoch ausnahmsweise gem. (\$ 44 i. V.m.) \31 Abs. 1 WHG zulässig sein. Dort ist beschrieben, wie zu verfahren ist, wenn lediglich vorübergehend eine Verschlechterung des Gewässerzustandes auftritt. ${ }^{85}$ Doch sind danach diese vorübergehenden $\mathrm{Zu}$ standsverschlechterungen $u$. a nur zulässig, wenn sie auf Umständen beruhen, die in natürlichen Ursachen begründet oder durch höhere Gewalt bedingt und die außergewöhnlich sind und nicht vorhersehbar waren oder durch Unfälle entstanden sind ( $\$ 31$ Abs. 1 Nr. 1 WHG). Munitionssprengungen sind aber weder von natürlichen Ursachen begründet oder durch höhere Gewalt bedingt, noch durch Unfälle entstanden. Demgegenüber kommt aber eine Ausnahme nach (\$44 i.V.m.) \$31 Abs. 2 S. 1 WHG in Betracht, da die Sprengungen regelmäßig eine Veränderung ,physischer Eigenschaften“ des Meeres, nämlich der Morphologie des Meeresbodens bewirken werden (\$31 Abs. 2 S. 1 Nr. 1 WHG); die Gründe dafür können zumindest von übergeordnetem öffentlichen Interesse sein (vgl. $\$ 31$ Abs. 2 S. 1 Nr. 2 WHG) und die mit den Sprengungen verfolgten Ziele werden wohl schwerlich mit anderen geeigneten, wesentlich geringere nachteilige Umweltauswirkungen zeitigenden Maßnahmen zu erreichen sein (vgl. \31 Abs. 2 S. 1 Nr. 3 WHG). Sofern zudem alle praktisch geeigneten Maßnahmen ergriffen werden, um die nachteiligen Auswirkungen der Sprengungen auf den Gewässerzustand zu verringern ( $\int 31$ Abs. 2 S. 1 Nr. 4 WHG), steht also einer wasserrechtlichen Ausnahme gemäß (\$ 44 i. V.m.) §31 Abs. 2 S. 1 WHG nichts im Wege.

74) Meyer-Teschendorf, JuS 1981, 187, 190.

75) Epping, in: ders./Hillgruber, BeckOK GG, 47. Ed. 15.5.2021, Art. 35 Rdnr. 8.

76) Gesetz zur Ordnung des Wasserhaushalts (Wasserhaushaltsgesetz - WHG) v. 31.7.2009, BGBl. I S. 2585; zuletzt geändert durch Art. 2 des Gesetzes v. 18. 8.2021, BGBl. I S. 3901.

77) Gesetz über Naturschutz und Landschaftspflege (Bundesnaturschutzgesetz - BNatSchG) v. 29.7.2009, BGBl. I S. 2542; zuletzt geändert durch Art. 1 des Gesetz v. 18.8.2021, BGB1. I S. 3908

78) Kotulla, WHG, 2. Aufl. 2011, $\$ 8$ Rdnr. 14.

79) Czychowski/Reinhardt, WHG, \$27 Rdnr. 14.

80) Meyer, in: Landmann/Rohmer, Umweltrecht, 95. EL Mai 2021, $\$ 47$ WHG Rdnr. 13

81) Kotulla, WHG, 2. Aufl. 2011, $\$ 27$ Rdnr. 5.

82) Verordnung zum Schutz der Oberflächengewässer v. 20.6.2016, BGBl. I S. 1373; zuletzt geändert durch Art. 2 Abs. 4 des Gesetzes v. 9.12.2020, BGBl. I S. 2873.

83) Durner, in: Landmann/Rohmer, UmweltR, WHG, 92. EL $2.2020, \$ 27$ Rdnr. 16.

84) Näheres zu den Schadstoffgrenzwerten in Czychowski/Reinhardt, in: Landmann/Rohmer, Umweltrecht, 95. EL Mai 2021, \$27 Rdnr. $11 \mathrm{ff}$

85) Kotulla, WHG, 2. Aufl. 2011, $\$ 31$ Rdnr. 3. 
In den Sprengungen muss also keine den Vorgaben des $\$ 12$ Abs. 1 WHG zuwiderlaufende Maßnahme gesehen werden. Die Erlaubniserteilung dafür wäre in Abhängigkeit vom jeweiligen Einzelfall und unter Berücksichtigung des Bewirtschaftungsermessen ( $\$ 12$ Abs. 2 WHG) durchaus möglich.

\subsubsection{2 $\int 44$ BNatSch G}

Ebenfalls aufgrund von $\$ 12$ Abs. 1 WHG muss sichergestellt sein, dass die Sprengungen nicht gegen Anforderungen nach anderen als (wasserhaushaltsrechtlichen) öffentlich-rechtlichen Vorschriften verstoßen. Hier kommen solche des Bundesnaturschutzgesetzes (BNatSchG) in Betracht. Denn gem. $\$ 56$ Abs. 1 BNatSchG gilt das BNatSchG auch im Bereich des Küstengewässers und im Wesentlichen ebenfalls innerhalb der AWZ. ${ }^{86}$ Dies schließt die im hiesigen Kontext besonders relevanten Regelungen zum Tötungs- und Verletzungsverbot gem. \44 BNatSchG ein. ${ }^{87}$ Gemäß $₫ 44$ Abs. 1 Nr. 1 BNatSchG ist es verboten, wildlebenden Tieren der besonders geschützten Arten nachzustellen, sie zu fangen, zu verletzen oder zu töten oder ihre Entwicklungsformen der Natur zu entnehmen, sie zu beschädigen oder sie zu zerstören. Folglich dürften die Munitionssprengungen diese besonders geschützten Arten nicht gefährden. Sowohl der Schweinswal (Phocoena phocoena) als auch der Seehund (Phoca vitulina) etwa gehören zu den gem. $\$ 7$ Abs. 2 Nr. 13 lit. b) bzw. Nr. 14 lit. b) BNatSchG i.V.m. dem Anh. IV der FaunaFlora-Habitat-Richtlinie (92/43/EWG - FFH-Richtlinie) vom 21.5.1992 ${ }^{88}$ besonders geschützten Tierarten. Gemäß $\int 44$ Abs. 1 Nr. 1 BNatSchG ist es u. a. verboten, wildlebende Tiere der besonders geschützten Arten zu verletzen oder zu töten. Es genügt die schlichte Tatsache des Verletzens oder Tötens zur Erfüllung des objektiven Tatbestands. ${ }^{89}$ Dabei ist es unerheblich, ob dies vorsätzlich, fahrlässig oder ohne Sorgfaltsverstoß unbeabsichtigt geschieht. ${ }^{90}$ Jedoch kann eine durch die Munitionssprengungen hervorgerufene Schädigung gem. $₫ 44$ Abs. 5 S. 2 Nr. 1 BNatSchG dann gerechtfertigt sein, wenn die Beeinträchtigung durch den Eingriff oder das Vorhaben das Tötungs- und Verletzungsrisiko für Exemplare der betroffenen Arten nicht signifikant erhöht und diese Beeinträchtigung bei Anwendung der gebotenen und fachlich anerkannten Schutzmaßnahmen nicht vermieden werden kann. ${ }^{91} \mathrm{Zu}$ klären ist zunächst, wann eine Maßnahme als unvermeidbar gilt. Dies soll nach $₫ 15$ Abs. $1 \mathrm{~S}$. 2 BNatSchG der Fall sein, wenn zumutbare Alternativen, den mit einem Eingriff verfolgten Zweck am gleichen Ort ohne oder mit geringeren Beeinträchtigungen von Natur und Landschaft $\mathrm{zu}$ erreichen, nicht gegeben sind. ${ }^{92} \mathrm{Ob}$ demnach eine Vermeidung der Beeinträchtigung möglich erscheint, ist unter Berücksichtigung der Belange der Gefahrenabwehr im jeweiligen Einzelfall abzuwägen. Für schwerwiegende Belastungen besonders schutzwürdiger Ökosysteme können durchaus bedeutsame Vermeidungsanstrengungen verlangt werden..$^{93}$

Eine fachlich anerkannte und zugleich aufwendige Schutzmaßnahme ist es, die Tiere durch laute Geräusche kurz vor der Sprengung zu verjagen. Auch sog. Blasenvorhänge (Big Bubble Curtain) können bei einer Sprengung zum Einsatz kommen. ${ }^{94}$ Hierfür wird unter Wasser mithilfe von Druckluft eine dichte Wand aus Luftbläschen erzeugt, die auf Detonationen wie ein Puffer wirkt und die Druck- und Schallwellen absorbieren soll. ${ }^{95} \mathrm{Als} \mathrm{ku}-$ mulative Tatbestandsvoraussetzung des $\$ 44$ Abs. 5 Nr. 1 BNatSchG darf sich das Tötungs- und Verletzungsrisiko für Exemplare der betroffenen Art zudem nicht signifikant erhöhen. Grundsätzlich sind Relativierungen des Tötungs- und Verletzungsverbots bezogen auf die Populationen der betreffenden Arten unzulässig. In der Praxis führt dies jedoch mitunter zu unüberwindbaren Hindernissen für die Planung und Durchführung von Vorhaben. ${ }^{96}$ Des- halb liegt nach der Rechtsprechung des BVerwG ein Verstoß gegen das Tötungs- und Verletzungsverbot nur dann vor, sofern dadurch das Risiko des Erfolgseintritts, d.h. das sozialadäquate Tötungs- und Verletzungsrisiko für die betreffenden Tiere signifikant erhöht würde. ${ }^{97}$ Dies ist der Fall, wenn etwa der Tod der Tiere über das bereits vorhabenunabhängige allgemeine Tötungsrisiko hinaus besteht. ${ }^{98}$ Ein Tötungsrisiko ergebe sich nicht nur aus dem allgemeinen Naturgeschehen, sondern sei auch dann sozialadäquat und deshalb hinzunehmen, wenn es zwar vom Menschen verursacht werde, aber nur einzelne Individuen und nicht die Population im Ganzen betreffe. ${ }^{99}$ Denn tierisches Leben existiere nicht in einer unberührten, sondern in einer vom Menschen gestalteten Landschaft. ${ }^{100}$ Dem Gericht zufolge greife nur innerhalb dieses Rahmens der Schutz des $₫ 44$ Abs. 1 Nr. 1 BNatSchG. Das bedeute aber nicht, dass gerade in einem Umfeld, in dem bereits wegen anderweitiger Vorbelastungen ein erhöhtes Tötungs- und Verletzungsrisiko bestehe, eine umso größere Gefährdung zulässig wäre. Umstände, die für die Beurteilung der Signifikanz eine Rolle spielen, sind vielmehr im Hinblick auf ihre artspezifischen Verhaltensweisen, der Häufigkeit der Frequentierung des durchschnittenen Raums und der Wirksamkeit vorgesehener Schutzmaßnahmen und - darüber hinaus - gegebenenfalls auch weiterer Kriterien im Zusammenhang mit der Biologie der Art zu bewerten. ${ }^{101}$ Der Erlaubniserteilung für die Sprengung von Munitionsaltlasten stünde somit auch $\ 44$ BNatSchG nicht unbedingt entgegen.

\subsubsection{Allgemeine Sorgfaltspflicht ( $\$ 5$ Abs. 1 Nr. 1 WHG)}

Insbesondere für den Fall der nach $₫ 8$ Abs. 2 S. 1 WHG erlaubnisfrei durchzuführenden Sprengung zur Abwehr einer gegenwärtigen Gefahr (oben 2.3.1) müssen jedenfalls die Bestimmungen des $\$ 5$ Abs. 1 WHG zur allgemeinen Sorgfaltspflicht beachtet werden. Danach $(§ 5$ Abs. 1 Nr. 1 WHG) ist jede Person verpflichtet, bei Maßnahmen, mit

86) Lütkes, in: ders./Ewer, BNatSchG, 2. Aufl. 2018, §56 Rdnr. 1-5; Fischer-Hüftle, NuR 2007, 78, $84 \mathrm{f}$.

87) Lüttgau, in: Giesberts/Reinhardt, BeckOK UmweltR, 59. Ed. 1.7.2021, \56 Rdnr. 7; Jenisch, NordÖR 2020, 493, 495.

88) AB1. 1992 L 206, S. 7.

89) Vgl. Stöckel/Müller-Walter, in: Erbs/Kohlhaas, Strafrechtliche Nebengesetze, BNatSchG, 236. EL 1.2020, \44 Rdnr. 10.

90) BT-Drs. 16/5100, S. 11.

91) Gellermann, in: Landmann/Rohmer, UmweltR, BNatSchG, 95. EL 5.2021, §44 Rdnr. 52.

92) Schrader, in: Giesberts/Reinhardt, BeckOK UmweltR, 59. Ed., 1.7.2021, \$15 Rdnr. 8.

93) Gellermann, in: Landmann/Rohmer, UmweltR, BNatSchG, 95. EL 5.2021, $\$ 15$ Rdnr. 5.

94) Antwort auf die Kleine Anfrage „Verwendung von Blasenschleiern beim Sprengen von Altmunition“, BT-Drs. 19/4511, S. 2 .

95) Diederichs/Pehlke/Nehls/Bellmann/Gerke/Oldeland/Grunau/ Witte/Rose, Schlussbericht: HYDROSCHALL-OFF BW II, Stand 21.9.2021, abrufbar unter https://bioconsult-sh.de/site/ assets/files/1312/1312.pdf, S. 35 ff.

96) Vgl. Gläß, in: Giesberts/Reinhardt, BeckOK UmweltR, 59. Ed 1.7.2021, $\int 44$ Rdnr. 16.

97) BVerwG, Beschl. v. 8.3.2018 - 9 B 25.17, NuR 2018, 625, 626.

98) BVerwG, Urt. v. 8.1.2014 - 9 A 4.13, NuR 2014, 413, 425 ; s.a. Gläß, in: Giesberts/Reinhardt, BeckOK UmweltR, 59. Ed. 1.7.2021, \44 Rdnr. 70a f.

99) BVerwG, Urt. v. 8.1.2014 - 9 A 4.13, NuR 2014, 413,434, BVerwG, Urt. v. 14.7.2011 - 9 A 12.10, NuR 2011, 866, 875; s. a. Gellermann, in: Landmann/Rohmer, UmweltR, BNatSchG, 95. EL 5.2021, \44 Rdnr. 9; a. A. lediglich OVG Münster Urt. v. 30.7.2009-8 A 2358/08, BeckRS 2010, 47328; VG Minden, Beschl. v. 10.3.2010 - 11 K 53/09, NuR 2010, 891, 894.

100) BVerwG, Beschl. v. 8.3.2018 - 9 B 25.17, NuR 2018, 625, 626.

101) BVerwG Beschl. v. 7.1.2020 - 4 B 20.19, BeckRS 2020, 1633 Rdnr. 5. 
denen Einwirkungen auf ein Gewässer verbunden sein können, die nach den Umständen erforderliche Sorgfalt anzuwenden, um eine nachteilige Veränderung der Gewässereigenschaften $\mathrm{zu}$ vermeiden. Auf diese Weise soll bereits die Entstehung der durch menschliches Verhalten bedingten Gefährdungen und Schädigungen abgewendet werden. ${ }^{102}$ Pflichtenadressat ist ,,jede Person“, also jede inoder ausländische natürliche und oder juristische Person; wozu Behörden und Körperschaften, Anstalten und Stiftungen des öffentlichen Rechts zählen. ${ }^{103}$ Somit richtet sich $₫ 5$ Abs. 1 WHG auch an die die Munitionsaltlasten beseitigenden Behörden. Mit den in Bezug genommenen Maßnahmen sind Handlungen jeder Art gemeint, mit denen Einwirkungen auf ein Gewässer verbunden sein können. ${ }^{104}$ Hierzu gehören auch Sprengungen von Munitionsaltlasten. Dementsprechend hat die jeweilige Behörde bei der Durchführung der Sprengungen die Sorgfalt anzuwenden, die nach objektiver Betrachtung im konkreten Einzelfall geboten ist. ${ }^{105}$ Dieser Sorgfaltsmaßstab richtet sich zunächst einmal nach den jeweils einschlägigen Spezialregelungen und/oder behördlich konkretisierten Vorgaben. Wenn es, wie im Falle der Munitionssprengungen, an solchen Regelungen und Vorgaben fehlt, muss die erforderliche Sorgfalt im Einzelfall nach objektiven Kriterien bestimmt werden. ${ }^{106}$ Dies hat zur Folge, dass der Pflichtenadressat alle tatsächlichen zur Pflichterfüllung erforderlichen Anstrengungen durchzuführen hat. An die Sorgfalt sind umso höhere Anforderungen zu stellen, je größer der infolge der Einwirkung auf das Gewässer drohende Schaden oder dessen Eintrittswahrscheinlichkeit ist. ${ }^{107}$ Die mit den Munitionsaltlastensprengungen verbundenen negativen Auswirkungen für die Flora und Fauna könnten den Eindruck erwecken, dass gegen das Schutzanliegen des $\ 5$ Abs. 1 Nr. 1 WHG verstoßen wird. Danach soll mit der Einhaltung des Sorgfaltsmaßstabes eine nachteilige Veränderung der Gewässereigenschaft möglichst vermieden werden. Eine solche Veränderung ist anzunehmen, sofern dadurch der Gebrauchswert des Gewässers für Menschen, Tiere und/oder Pflanzen herabgesetzt wird. ${ }^{108}$ Dieser Nachteil wird vermieden, wenn Sorge dafür getragen ist, dass die nachteilige Veränderung nach menschlichem Dafürhalten nicht eintritt oder aber - soweit bereits eingetreten - zumindest nicht weiter anwächst. ${ }^{109}$ Aus dieser „Schadenminderungsobliegenheit" ${ }^{110}$ wird geschlussfolgert, dass z.B. im Falle eines Tankwagenunfalls, bei dem wassergefährdende Flüssigkeit aus dem Tank ausgelaufen und in den Boden eingedrungen ist und weiteres Auslaufen und eine weitere Versickerung in den Boden droht, diejenigen Maßnahmen $\mathrm{zu}$ ergreifen sind, die ein weiteres Auslaufen verhindern (Verschließen des Tanks, Umfüllen des Tankinhalts) und ein weiteres Eindringen der Flüssigkeit in den Boden zu verhindern (Ausbaggern und Fortschaffen des verseuchten Erdreichs). ${ }^{111}$ Die Sprengungen von Munitionsaltlasten führen für die Gewässerökologie zwar regelmäßig zu erhebliche Schäden, jedoch wird der latente Schaden für die Meeresumwelt, welcher durch das einfache Vorhandensein der Munitionsaltlasten im Gewässer entsteht, beendet. Deshalb vermag ein kurzer Eingriff in die Meeresökologie auch dem Gebot des $₫ 5$ Abs. 1 Nr. 1 WHG standzuhalten, sofern es an einer gewässerschonenderen Beseitigungsalternative mangelt.

\section{Schlussbetrachtung}

Die vorangegangenen Ausführungen verdeutlichen, dass die schon gefahrenabwehrrechtliche Kompetenzzuordnung zwischen Bund und Ländern bei der Beseitigung von Munitionsaltlasten sehr komplex ist. Das kann negative Auswirkun- gen auf die Effektivität der zu treffenden Gefahrenabwehrmaßnahmen haben und führt überdies zur Rechtsunklarheit auf Seiten der Behörden. Dies ist nicht zuletzt der föderalen Kompetenzverteilung und den nur sehr begrenzten nautischen Bundeskompetenzen geschuldet. Ein koordiniertes Vorgehen zwischen den verschiedenen Behörden dürfte unverzichtbar sein. Die Frage, ob die Bundeswehr den zivilen Behörden gemäß Art. 35 Abs. 1 GG Amtshilfe leisten kann, ist grundsätzlich zu bejahen, wenn ein entsprechendes Hilfegesuch der Zivilbehörde vorliegt.

Jedoch sind bei den durchzuführenden Gefahrenabwehrmaßnahmen jedenfalls die Anforderungen des Gewässerund Naturschutzrechts zu beachten. Im Übrigen konnte aufgezeigt werden, dass das Gewässerschutz- und Naturschutzrecht der Vornahme der Sprengung von Munitionsaltlasten gerade in den konkreten Fällen nicht entgegensteht, in denen die Sprengung zur Abwehr gegenwärtiger Gefahren erforderlich ist. Liegt eine solche gegenwärtige Gefahrsituation nicht vor, sind die Sprengungen indes gem. os 8 Abs. 1, 9 Abs. 2 Nr. 2 WHG erlaubnispflichtig und in engen wasser- wie naturschutzrechtlichen Grenzen auch erlaubnisfähig. Ungeachtet dessen bleibt jedoch stets zu beachten, dass gerade wegen der nach wie vor latent toxischen wie explosiven Eigenschaften der maritim abgelagerten Altmunition die Hinauszögerung ihrer Beseitigung fatale Auswirkungen auf die Meeresumwelt hat bzw. haben kann, da diesenfalls weiterhin die toxischen Inhaltsstoffe der Altmunition in die Meeresumwelt freigesetzt werden. ${ }^{12}$

Open Access. Dieser Artikel wird unter der Creative Commons Namensnennung 4.0 International Lizenz veröffentlicht, welche die Nutzung, Vervielfältigung, Bearbeitung, Verbreitung und Wiedergabe in jeglichem Medium und Format erlaubt, sofern Sie den/die ursprünglichen Autor(en) und die Quelle ordnungsgemäß nennen, einen Link zur Creative Commons Lizenz beifügen und angeben, ob Änderungen vorgenommen wurden.

Die in diesem Artikel enthaltenen Bilder und sonstiges Drittmaterial unterliegen ebenfalls der genannten Creative Commons Lizenz, sofern sich aus der Abbildungslegende nichts anderes ergibt. Sofern das betreffende Material nicht unter der genannten Creative Commons Lizenz steht und die betreffende Handlung nicht nach gesetzlichen Vorschriften erlaubt ist, ist für die oben aufgeführten Weiterverwendungen des Materials die Einwilligung des jeweiligen Rechteinhabers einzuholen.

Weitere Details zur Lizenz entnehmen Sie bitte der Lizenzinformation auf http://creativecommons.org/licenses/by/4.0/deed.de.

Open Access funding enabled and organized by Projekt DEAL.

102) Kotulla, WHG, 2. Aufl. 2011, \$5 Rdnr. 2

103) Kotulla, WHG, 2. Aufl. 2011, \$5 Rdnr. 8.

104) Hasche, in: Giesberts/Reinhardt, BeckOK WHG, 55. Ed. 1.12.2017, \$5 Rdnr. 4

105) Hasche, in: Giesberts/Reinhardt, BeckOK WHG, 55. Ed. 1. 12.2017, §5 Rdnr. 5.

106) Faßbender, in: Landmann/Rohmer, UmweltR, WHG, 95. EL 5.2021, §5 Rdnr. 17

107) Kotulla, WHG, 2. Aufl. 2011, \$ 5 Rdnr. 10.

108) BGH, Urt. v. 21.1.1988 - III ZR 180/86, NuR 1988, $202 \mathrm{ff}$

109) Kotulla, WHG, 2. Aufl. 2011, \5 Rdnr. 12.

110) Kotulla, WHG, 2. Aufl. 2011, \5 Rdnr. 12.

111) Knopp, in: Sieder/Zeitler/Dahme/ders., 55. EL 9.2020, WHG $\$ 5$ Rdnr. 38

112) Arbeitsgemeinschaft „Rüstungsaltlasten im Meer“: Ökotoxikologisches Gefahrenpotential durch sprengstofftypische Verbindungen und Tabun aus Munitionsaltlasten in der schleswig-holsteinischen Nord- und Ostsee, Stand 21.9.2021, abrufbar unter https://www.schleswig-holstein.de/DE/ Fachinhalte/M/meeresschutz/Downloads/Burmeier_Studie. pdf?__blob $=$ publicationFile $\& v=1$, S. $235 \mathrm{ff}$. 